\title{
Thyroid hormones and heart mitochondrial nitric oxide under hypovolemia
}

\begin{abstract}
The aim of the present work was to examine the effect of thyroid state on rat heart mitochondria function during hypovolemia. Sprague-Dawley rats treated with $\mathrm{T}_{3}$ (hyper, $20 \mu \mathrm{g} / 100 \mathrm{~g}$ body weight) or $0.02 \%$ methimazole (hypo, w/v) for 28 days. Hypovolemia was induced by acute hemorrhage. $\mathrm{O} 2$ uptake, complex I activity and mitochondrial nitric oxide synthase (mtNOS) protein levels were determined in heart mitochondria. The malate-glutamate-supported state 3 respiration decreased and increased in hypo and hyperthyroid rats, while state 4 respirations did not change. Complex I activity and mtNOS protein levels were decreased in hypothyroid rats even in acute hypovolemia. Hyperthyroidism did not change these parameters. In summary, this study showed, for the first time, interesting findings that heart mitochondrial function is altered during thyroid disorder and acute hypovolemia. Heart mitochondrial nitric oxide, modulating oxygen uptake, may be involved in the adaptative response to assure the cardiomyocytes survives in this experimental condition.
\end{abstract}

Keywords: thyroid gland, cardiomyocytes, oxygen uptake, mtNOS, complex I
Volume 6 Issue 5 - 2018

\author{
Natalia Ogonowski,' Laura Beatriz Valdez, \\ Tamara Zaobornyj,, María Bernardita \\ Puchulu, ${ }^{2}$ Ana María Balaszczuk, ${ }^{2}$ Andrea \\ Lorena Fellet ${ }^{2}$ \\ 'Cátedra de Fisiología, Facultad de Farmacia y Bioquímica, \\ Universidad de Buenos Aires, Instituto de Química y \\ Metabolismo del Fármaco (IQUIMEFA), Argentina \\ ${ }^{2}$ Cátedra de Fisicoquímica, Facultad de Farmacia y Bioquímica, \\ Universidad de Buenos Aires, Instituto de Bioquímica y Medicina \\ Molecular (IBIMOL), Argentina
}

Correspondence: Natalia Ogonowski Cátedra de Fisiología, Facultad de Farmacia y Bioquímica, Universidad de Buenos Aires, Instituto de Química y Metabolismo del Fármaco (IOUIMEFA), CONICET, I I I 3 Buenos Aires, Argentina, Tel +54 II 52874709, Fax+54 II 52874709, Email nogonowski@ffyb.uba.ar

Received: July 30, 2018 | Published: September 21, 2018

\section{Introduction}

The thyroid hormones are known as one of the major factors that regulate cardiac metabolic and physiological functions ${ }^{1}$ Mitochondria is considered a subcellular target of thyroid hormone action and a major compartment of this hormone accumulation ${ }^{2,3}$ In recent years, nitric oxide (NO) signaling has been identified as an important trigger of cardioprotection in the mitochondria ${ }^{4}$. It is synthesized from $\mathrm{L}$-arginine, $\mathrm{NADPH}$ and $\mathrm{O}_{2}$ in a reaction catalyzed by mitochondria NO synthase (mtNOS) isoform located in the mitochondrial inner membrane. ${ }^{5,6}$ The mtNOS is the $\alpha$ splice variant of neuronal nitric oxide synthetase isoform resulting in a novel $\mathrm{Ca}^{+2}$ dependent NOS subtyped. ${ }^{7,8}$ Thyroid hormones modulate NO steady-state level which may act as a messenger to modulate the mitochondrial bioenergetic function. ${ }^{9,10}$ In this context, NO modulates cellular respiration acting on the complexes of respiratory chain. ${ }^{11,12}$ It is well known that hypovolemia may lead sequentially to hemodynamic instability, decreased in oxygen delivery, tissue perfusion and cellular hypoxia. ${ }^{13}$ The pathogenesis of organ injury secondary to this acute stress is still not completely understood. Experimental studies of our laboratory indicate that hypovolemic state induced by acute hemorrhage triggered a heterogeneous and dynamic NOS activation, modulating the cardiovascular response. Involvement of the NO pathway occurs in an isoform-specific and time-dependent manner. ${ }^{14}$ However, the exact mechanism underlying the effects of the thyroid hormones on heart mitochondrial NO production against acute hemorrhage is still unknown. Considering the mitochondria as an important target for thyroid hormone action and a crucial organelle associated with metabolic process related to cell survive in different pathological situations, we decided to examine rat heart mitochondrial function during thyroid disorder and hypovolemia. We also evaluate the role of $\mathrm{NO}$ as a probable messenger that underlines the actions of thyroid hormone at the mitochondrial level.

\section{Materials and methods}

\section{Animals}

Male Sprague-Dawley rats of 2 months old from the breeding laboratories of the School of Pharmacy and Biochemistry (Universidad de Buenos Aires, Argentina) were used throughout the study. Rats were housed in humidity- and temperature-controlled environment with an automatic $12 \mathrm{~h}$ light: $12 \mathrm{~h}$ darkness cycle. All procedures were reviewed and approved by the National Food, Drug and Medical Technology Administration (ANMAT), National Department of Health and Environment, Argentina (No. 6344/96) and CICUAL N ${ }^{\circ}$ 0054570. Rats were randomly assigned to one of the three groups:

Control rats (Eut, $\mathrm{n}=16$ ), animals who received i.p injections of $0.9 \% \mathrm{NaCl}(0.1 \mathrm{ml} / 100 \mathrm{~g}$ body weight $)$ every $2^{\text {nd }}$ day for 28 days.

$T_{3}$-treated rats (Hyper, $\mathrm{n}=16$ ), animals received i.p injections of triiodothyronine $\left(\mathrm{T}_{3}\right)$ (Sigma, $20 \mathrm{ug} / 100 \mathrm{~g}$ body weight) every $2^{\text {nd }}$ day for 28 days. $^{15}$

Methimazole-treated rats (hypo, $\mathrm{n}=16$ ), animals were rendered hypothyroid after 28 days of treatment with $0.02 \%$ methimazole (w/v) in the drinking water. ${ }^{16}$

\section{Determination of treatment efficacy}

Serum thyroid-stimulating hormone (TSH), total $\mathrm{T}_{3}$ and thyroxin $\left(\mathrm{T}_{4}\right)$ (TSH kit, National Institute of Diabetes and Digestive and Kidney Diseases, National Institutes of Health, Bethesda, USA) were measured by radioimmunoassay at the beginning and the end of the experimental period. ${ }^{17}$

\section{Hemodynamic parameters}

After 28 days of treatment, the animals were anesthetized with urethane $(1 \mathrm{~g} / \mathrm{kg}$ ip). A tracheotomy was performed using polyethylene 
tubing (4mm ID, Portex). Mean arterial pressure (MAP) was measured through a cannula inserted into the right femoral artery and connected to a pressure transducer (Statham P23 ID, Gould Inst, Cleveland, OH, USA). Measurements were recorded with a polygraph (Physiograph E \& M, Houston, TX, USA) during the whole experiment. Heart rate (HR) was determined from the pulsatile pressure signal by beat-to-beat conversion with a tachograph amplifier (model S7726 tachometer, Coulbourn Instruments, Allentown, PA, USA). The Labtech Notebook program (Laboratory Technology, Wilmington, MD, USA) was used for data acquisition.

\section{Experimental protocol}

Eut, Hyper and hypo animals were subdivided into two experimental groups:

1) Control rats (C). After a 30-min stabilization period, MAP and HR were continuously recorded over a 120 -min period. $(n=8$ each group).

2) Hemorrhaged rats $(H)$. After a 30-min stabilization period, basal MAP and HR were measured over a 5-min period. Subsequently, the acute hemorrhage was performed. Thereafter, hemodynamic parameters were continuously recorded over a 120-min period after the bleeding ( $\mathrm{n}=8$ each group). The hypovolemic state in $H$ was induced through an acute hemorrhage using a cannula inserted in the left femoral artery (Riviero, PR10). The bleeding was done by a loss of $20 \%$ of the blood volume for $2 \mathrm{~min}$. The volume of blood loss was calculated individually for every animal, from the total blood volume ( $7 \%$ of the body mass).

\section{Heart mitochondrial isolation}

Heart mitochondria were obtained from tissue homogenates by differential centrifugation (Sorvall- Instruments-Du Pont, Model RC5S, Buckinghamshire, England) in an ice-cold medium (1/10) containing $230 \mathrm{mM}$ mannitol, $70 \mathrm{mM}$ sucrose, $1 \mathrm{mM}$ EDTA, $10 \mathrm{mM}$ Tris-HCl, pH 7.4 (MSTE) for $15 \mathrm{~s}$ with a blade homogenizer (KendroSorvall-Du Pont Inst., Asheville, NC, USA) and by five strokes in a glass-Teflon homogenizer. The homogenates were centrifuged at $600 \mathrm{~g}$ for $10 \mathrm{~min}$ to discard nuclei and cell debris and the supernatant was centrifuged at $8000 \mathrm{~g}$ for $10 \mathrm{~min}$ to precipitate mitochondria, which were washed with MSTE. All procedure was carried out at 0-4 ${ }^{\circ} \mathrm{C}$. Protein concentrations were determined with the Folin reagent, using bovine serum albumin (BSA) as standard. ${ }^{18}$

\section{Mitochondrial $\mathrm{O}_{2}$ consumption}

Mitochondrial $\mathrm{O}_{2}$ uptake was measured polarographically with a Clark-type electrode (Hansatech Oxygraph, Hansatech Instruments Ltd, Norfolk, England). State 4 respiration was determined at $30^{\circ} \mathrm{C}$ in an air-saturated $\left(220 \mu \mathrm{M} \mathrm{O}_{2}\right)$ reaction medium consisting of $120 \mathrm{mM}$ $\mathrm{KCl}, 5 \mathrm{mM} \mathrm{KH}_{2} \mathrm{PO}_{4}, 1 \mathrm{mM}$ EGTA, $3 \mathrm{mM}$ HEPES, $1 \mathrm{mg} \mathrm{ml}^{-1} \mathrm{BSA}, 2$ $\mathrm{mM}$ malate and $5 \mathrm{mM}$ glutamate (complex I substrates), $\mathrm{pH} 7.20$, and heart mitochondrial suspension $(0.1-0.3 \mathrm{mg}$ mitochondrial protein $\mathrm{ml}^{-1}$ ). State $3 \mathrm{O}_{2}$ consumption rate was established by supplementation of this medium with $0.5 \mathrm{mM} \mathrm{ADP}{ }^{19}$. Respiration was expressed in ng-at $\mathrm{O} / \mathrm{min}$. mg protein, and respiratory control was calculated as the ratio of state $3 /$ state 4 respiration rates.

\section{Complex I activity}

The enzyme activity of complex I was determined spectrophotometrically (Beckman DU 7400 spectrophotometers; 550 $\mathrm{nm}, \varepsilon=19 \mathrm{mM}^{-1} \mathrm{~cm}^{-1}$ ) as NADH-cytochrome c reductase at $30^{\circ} \mathrm{C}$ with mitochondrial membranes suspended in $100 \mathrm{mM} \mathrm{KH_{2 }} \mathrm{PO}_{4} / \mathrm{K}_{2} \mathrm{HPO}_{4}$,
$\mathrm{pH}$ 7.4. Mitochondrial membranes were added with $0.2 \mathrm{mM} \mathrm{NADH}$, $25 \mu \mathrm{M}$ cytochrome $\mathrm{c}^{3+}$, and $0.5 \mathrm{mM} \mathrm{KCN}$. Enzymatic activities were expressed as nmol reduced cytochrome c/min. mg protein. ${ }^{20}$

\section{Western blot analysis}

The proteins of mitochondrial membranes $(0.10 \mathrm{mg}$ protein/lane $)$ were separated by electrophoresis in 7.5\% SDS-polyacrylamide gels and transferred to a nitrocellulose membrane (Bio-Rad, Munich, Germany) and then incubated with rabbit polyclonal anti-neuronal NOS (BD Transduction Laboratories, USA) and anti-VDAC-1/2/3 (FL-283: sc-98708, Santa Cruz Biotechnology, CA) antibodies [1:500 dilution] and a horseradish peroxidase-conjugated goat anti-rabbit secondary antibody (1:5,000 dilution). Samples were revealed by chemiluminescence using the enhanced chemiluminescence reagent (Amersham Pharmacia Biotechnology, Uppsala, Sweden) for 4 min. Quantification of the bands was performed by digital image analysis using a Hewlett-Packard scanner and Totallab analyzer software (Biodynamics, Seattle, WA). Data were expressed as relative to VDAC-1/2/3 expression (loading control). All experiments were performed in triplicate. ${ }^{15}$

\section{Statistical analysis}

Data in tables and figures are mean values \pm SEM. Data were evaluated with univariate and multivariate approaches for a completely randomized design, with a structure of two factors (hemorrhage and thyroid hormones). For each variable, ANOVA or MANOVA analysis was performed when appropriate. The Levene's and Shapiro-Wilk's tests were used to evaluate homogeneity of variances and normality of data, respectively. When normality and homogeneity of variances assumptions were satisfied, the Bonferroni multiple comparison test was run. In the case of non-homogenous variances, a multiple comparison test, such as Tamhane, was run. To detect association among variables, a correlation analysis was performed and the Pearson coefficient was calculated. All statistical procedures were performed using the SPSS statistical software package version 22.0 statistical significance was set at $\mathrm{P}<0.05$.

\section{Results}

\section{Treatment efficacy}

TSH plasmatic levels were higher and lower in hypo and Hyper rats respectively. $T_{3}$ and $T_{4}$ levels decreased in hypo rats, while $T_{4}$ increased in Hyper animals. Body weights and basal MAP values were similar in the three groups of animals. However, basal HR values were lower and higher in hypo and Hyper rats compared with Eut animals, respectively. Bleeding induced a decreased of MAP values in all experimental groups. However, animals with thyroid disorder exhibited a lower MAP values at 120 minutes of hemorrhage than Eut rats (Table 1).

\section{Mitochondrial respiratory function}

The malate-glutamate-supported state 3 respiration was decreased in hypo rats and increased in hyper rats. Hemorrhage did not change state 3 respiration in all groups of animals. No differences were observed in malate-glutamate-supported state 4 between experimental groups. Withdrawal did not modify this state (Table 2). Respiratory control and ADP-to-O ratios were high indicating that the mitochondria were well coupled and able to effectively carry out oxidative phosphorylation. After bleeding, it was observed a tendency of ADP-to-O ratio to decrease in hypo animals (Table 2). 
Table I Biological variables

\begin{tabular}{|c|c|c|c|}
\hline Animals & Eut & Hypo & Hyper \\
\hline $\mathrm{TSH}(\mathrm{ng} / \mathrm{ml})$ & $14.75 \pm 0.83$ & $35.57 \pm 4.35 *$ & $5.57 \pm 0.03 *$ \\
\hline T3 (ng/dl) & $1.131 \pm 0.123$ & $0.750 \pm 0.036 *$ & $1.034 \pm 0.036$ \\
\hline T4 (ug/ml) & $2.475 \pm 0.031$ & $1.034 \pm 0.036^{*}$ & $3.775 \pm 0.270^{*}$ \\
\hline BW (g) & $337 \pm 12$ & $338 \pm 12$ & $298 \pm 12$ \\
\hline Basal HR (bpm) & $352 \pm 15$ & $214 \pm 13^{*}$ & $424 \pm 15^{*}$ \\
\hline At 120 min HR (bpm) & $364 \pm 12$ & $222 \pm 10 *$ & $480 \pm 10 *$ \\
\hline Basal MAP(mmHg) & $80 \pm 4$ & $70 \pm 4$ & $76 \pm 2$ \\
\hline At $120 \mathrm{~min}$ MAP $(\mathrm{mmHg})$ & $58 \pm 3$ & $45 \pm 2 *$ & $41 \pm 2 *$ \\
\hline LVIDd (mm) & $5.40 \pm 0.18$ & $5.99 \pm 0.19 *$ & $4.7 \pm 0.10^{*}$ \\
\hline LVIDs (mm) & $2.80 \pm 0.06$ & $3.01 \pm 0.10 *$ & $2.2 \mathrm{I} \pm 0.07^{*}$ \\
\hline $\mathrm{EF}(\%)$ & $86 \pm 3$ & $83 \pm I^{*}$ & $88 \pm 3$ \\
\hline FS (\%) & $56 \pm 2$ & $46 \pm 2 *$ & $51 \pm 2$ \\
\hline
\end{tabular}

Eut, Euthyroid rats; hypo, Hypothyroid rats; Hyper, Hyperthyroid rats; TSH, Thyroid-stimulating hormone; T3 Triiodothyronine; T4, Total thyroxine; BW, Body weight; HR, Heart rate; MAP, Mean arterial pressure; LVIDd LV internal diameter in diastole; LVIDs, LV internal diameter in systole; EF, Ejection fraction; FS, Fractional shortening. Data are mean $\pm \mathrm{SEM} ; \mathrm{n}=15 ; * \mathrm{P}<0.05$ vs Eut rats.

Table 2 Heart mitochondria function in thyroid disorder and hypovolemia

$\mathrm{O}_{2}$ Uptake, ng-atoms $O$ min - Img protein-I

\begin{tabular}{lllll}
\hline Malate-glutamate & State 4 & State 3 & $\begin{array}{l}\text { Respiratory } \\
\text { control ratio }\end{array}$ & ADP-to-O ratio \\
\cline { 2 - 5 } Eut & $42 \pm 3$ & $216 \pm 13$ & 5.2 & $2.50 \pm 0.08$ \\
EutH & $45 \pm 3$ & $228 \pm 10$ & 5.1 & $2.49 \pm 0.17$ \\
hypo & $35 \pm 2$ & $177 \pm 7^{*}$ & 5.1 & $2.54 \pm 0.19$ \\
hypoH & $40 \pm 2$ & $174 \pm 4$ & 4.3 & $2.13 \pm 0.06$ \\
Hyper & $40 \pm 2$ & $260 \pm 14^{*}$ & 6.5 & $2.54 \pm 0.09$ \\
HyperH & $42 \pm 2$ & $257 \pm 13$ & 6.1 & $2.57 \pm 0.23$
\end{tabular}

Eut, Euthyroid rats; hypo, Hypothyroid rats; Hyper, Hyperthyroid rats; H, Hemorrhaged. Data are mean $\pm \mathrm{SEM}$; $\mathrm{n}=\mathrm{I}$; $* \mathrm{P}<0.05$ vs Eut rats.

\section{Complex I activity}

Enzyme activity was only decreased in hypo rats. This activity was increased after bleeding in hypo animals. The complex I activity did not change in Hyper animals and the bleeding did not change this parameter (Table 3). 
Table 3 Complex I activity in heart mitochondria in thyroid disorder and hypovolemia

\begin{tabular}{ll}
\hline Animals & Complex I (nmol/min .mg protein) \\
\hline Eut & $204 \pm 44$ \\
Eut H & $182 \pm 18$ \\
hypo & $117 \pm 16^{*}$ \\
hypo H & $147 \pm 17 \dagger$ \\
Hyper & $179 \pm 14$ \\
Hyper H & $179 \pm 10$ \\
\hline
\end{tabular}

Eut, Euthyroid rats; hypo, Hypothyroid rats; Hyper, Hyperthyroid rats; $\mathrm{H}$, Hemorrhaged. Data are mean \pm SEM; $n=15 ; * P<0.05$ vs Eut rats; † $P<0.05$ vs rats without hemorrhage.

\section{Nitric oxide synthase in heart mitochondria}

Figure 1 showed that hypothyroidism decreased nNOS protein levels compared with Eut group. This protein levels did not change in Hyper animals. Withdrawal decreased nNOS protein levels in hypo and Eut rats, while did not change in Hyper animals.

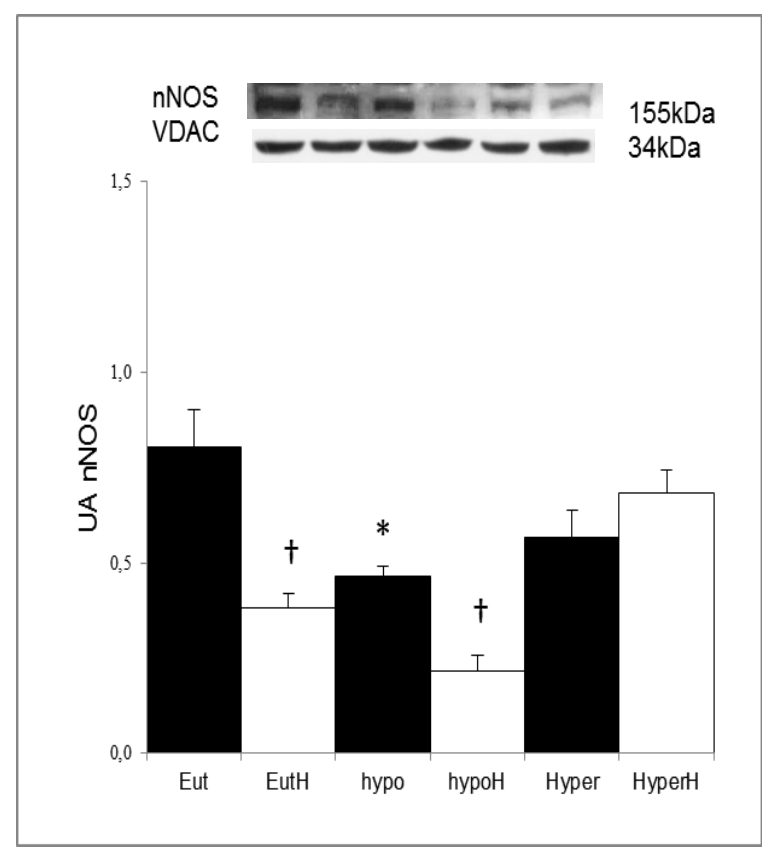

Figure 1 Representative Western Blots of nNOS levels, carried out on proteins from euthyroid (Eut), hypothyroid (hypo) and Hyperthyroid (Hyper) in heart mitochondria. The values are mean $\pm \mathrm{SEM} ; \mathrm{n}=15$ /group; $* \mathrm{P}<0.05$ vs Eut rats; $\dagger \mathrm{P}<0.05$ vs rats without hemorrhage. Histograms illustrate the mean NOS protein values for each group. All experiments were performed in triplicate. Each blot was normalized with the expression of the VDAC from the same gels. Data are mean \pm SEM; $\mathrm{n}=7 /$ group; $* \mathrm{P}<0.05$ vs Eut ats; $\uparrow \mathrm{P}<0.05$ vs rats without hemorrhage. EutH: euthyroid hemorrhaged rats; hypoH: hypothyroid hemorrhaged rats; HyperH: hyperthyroid hemorrhaged rats.

\section{Discussion}

This study showed, for the first time, interesting findings that heart mitochondrial function is altered during thyroid disorder and acute hypovolemia. Heart mitochondrial NO, modulating oxygen uptake, may be involved in the adaptative response to assure the cardiomyocytes survives in this experimental condition.

Several authors show that thyroid hormones have a special role regulating heart function. In this context, cardiovascular system regulation would be crucial in these pathological situations of hypovolemia. In this study, TSH measurements showed that both treatments were effective to establish thyroid disorder. Basal MAP values were similar in all experimental groups of rats. These findings were like those obtained by us previously and were discussed previously. ${ }^{21}$ The hemodynamics changes let us to evaluate the mitochondrial function in the experimental groups. The determination of this function was achieved by measuring $\mathrm{O}_{2}$ consumption in presence of saturating concentrations of the respective substrates. ${ }^{22}$ Thyroid state only affects state 3 of respiration using malate-glutamate as a substrate, while no changes were observed in oxygen consumption during state 4 . Additionally, it is important to note that oxygen uptakes did not change in hypovolemic induced-acute cellular stress. Complex I activity decreased only in hypothyroidism. This finding agreed with those found by several authors. ${ }^{16-23}$ This effect could be probably controlled by a variety of mechanisms such as reduced content and activity of some components of the electron transport chain, alterations of mitochondrial membranes permeability as well as changes in lipid composition. ${ }^{23,24}$ Additionally, Mukherjee et al., ${ }^{25}$ showed that complex I activity was inhibited in hypothyroid submitochondrial particles, whereas $T_{3}$ supplementation upregulated electron transport chain complexes. Although it is well known that $T_{3}$ upregulates all the respiratory chain complexes of inner mitochondrial membrane accounting for enhanced oxygen consumption as it was shown by our results. On the other hand, we observed that hemorrhage decreased this complex I activity only in hypo group. In accordance with our results, we suggest that complex I seems to be a vulnerable target in hypothyroid condition. While several factors may be responsible of this effect, we focused on mitochondrial NO production. The results showed that mtNOS protein levels only decreased in hypo animals compared with Eut rats. This result would be disagreed with several authors who showed in other tissues like liver and skeletal muscle, an increased mtNOS expression in hypothyroidism. ${ }^{16-26}$ That is, hypothyroidism is associated with a lower activity of complex I and lower mtNOS protein level in mitochondrial heart compared with Eut animals. Parihar et al. showed a similar result suggesting that rat liver and brain $\mathrm{mtNOS}$ are functionally associated with mitochondrial respiration chain complex I. ${ }^{27}$ Additionally, our study also showed that this interaction would depend on thyroid hormone levels. Interestingly, mitochondrial dysfunction is often associated with critical care diseases such as sepsis, trauma and acute hemorrhagic. ${ }^{28}$ The results show that in our experimental conditions the hypovolemia induced by acute bleeding did not modify the oxygen consumption in any of the three groups of animals. On the other hand, we also show that the maintenance of normal respiration after bleeding was associated with a decrease in the levels of mtNOS only in Eut and hypothyroid animals. These findings suggest that mtNOS would be related to energy demand and it reacts differently depending thyroid state and volemia.

\section{Conclusion}

The data presented in this study provide the following novel information: heart mitochondrial function is altered during thyroid disorder and acute hypovolemia. The decrease of thyroid hormone level would probably be a hormonal environment that promotes 
changes in mitochondrial NO bioavailability modulating oxygen consumption and cell respiration even against acute hypovolemic stress. Alterations of complex I activity could mediate these effects.

\section{Acknowledgements}

None

\section{Conflict of interest}

The author declares there is no conflict of interest.

\section{References}

1. Klein I Danzi S. Thyroid disease and the heart. Curr Probl Cardiol. 2016; Feb 41(2):65-92.

2. Davis PJ Goglia F, Leonard JL. Nongenomic actions of thyroid hormone Nat Rev Endocrinol. 2016; 12: 111-21.

3. Harper ME, Seifert EL. Thyroid Hormone Effects on Mitochondrial Energetics. Thyroid. 2008; 18: 145-56.

4. Dongworth RK, Hall Ar, Burke N, et all. Targeting mitochondria for cardio protection: examining the benefit for patients. Future Cardiol. 2014; 10 (2): $255-72$

5. Ghafourifar P, Cadenas E. Mitochondrial nitric oxide synthase. Trends Pharmacol Sci. 2005; 26: 190-5.

6. Giulivi C, Poderoso JJ, Boveris A. Production of nitric oxide by mitochondria. J Biol Chem. 1998; 273: 11038-43.

7. Ghafourifar P, Richter C. Nitric oxide synthase activity in mitochondria FEBS Lett. 1997; 418: 291-296.

8. Lacza Z, Snipes JA, Zhang J, et all. Mitochondrial nitric oxide synthase is not eNOS, nNOS or iNOS. Free Radic Biol Med; 2003 35: 1217-28.

9. Fellet AL, Arza P, Arreche N, Arranz C, Balaszczuk AM. Nitric oxide and thyroid gland: modulation of cardiovascular function in autonomicblocked anaesthetized rats. Exp Physiol. 2004; 89: 303-12.

10. Fellet AL, Balaszczuk AM, Arranz C, et all. Autonomic regulation of pacemaker activity: role of heart nitric oxide synthases. Am J Physiol Heart Circ Physiol. 2006; 291: H1246-54.

11. Moncada S, Erusalimsky JD. Does nitric oxide modulate mitochondrial energy generation and apoptosis? Nat Rev Mol Cell. 2002; 3: 214-20.

12. Xu F, Qiao S, Li H, et all. The Effect of Mitochondrial Complex I-Linked Respiration by Isoflurane Is Independent of Mitochondrial Nitric Oxide Production. Cardiorenal Med. 2018; 8: 11322 .

13. Zenebe WJ, Nazarewicz RR, Parihar MS, et all. Hypoxia/reoxygenation of isolated rat heart mitochondria causes cytochrome $\mathrm{c}$ release and oxidative stress; evidence for involvement of mitochondrial nitric oxide synthase. $J$ Mol Cell Cardiol. 2007; 43: 411-9.

14. Balaszczuk AM, Arreche ND, Mc Laughlin M, et all. Nitric oxide synthases are involved in the modulation of cardiovascular adaptation in hemorrhaged rats. Vascul Pharmacol. 2006; 44: 417-26.
15. Heron MI, Rakusan K. Short- and long-term effects of neonatal hypo- and hyperthyroidism on coronary arterioles in rat. Am J Physiol. 1996; 271 : H1746-54.

16. Franco MC, Antico Arciuch VG, Peralta JG, et all. Hypothyroid phenotype is contributed by mitochondrial complex I inactivation due to translocated neuronal nitric-oxide synthase. J Biol Chem. 2006; 281: 4779-86.

17. Greeley GH Jr, Lipton MA, Kizer JS. Serum thyroxine, triiodothyronine and TSH levels and TSH release after TRH in aging male and female rats. Endocr Res Commun. 1982; 9: 169-77.

18. Iglesias DE, Bombicino SS, Valdez LB, et all. Nitric oxide interacts with mitochondrial complex III producing antimycin-like effects. Free Radic Biol Med. 2015; 89: 602-13.

19. Boveris A, Costa LE, Cadenas E, Poderoso JJ. Regulation of mitochondrial respiration by adenosine diphosphate, oxygen and nitric oxide. Methods Enzymol. 1999; 301:188-198.

20. Zaobornyj T, Valdez LB, Iglesias DE, et all. Mitochondrial nitric oxide metabolism during rat heart adaptation to high altitude: effect of sildenafil, L-NAME and L-arginine treatments. Am J Physiol Heart Circ Physiol. 2009; 296: 1741-7.

21 Ogonowski N, Piro G, Pessah D, et all. Thyroid disorders and nitric oxide in cardiovascular adaptation to hypovolemia. J Endocrinol. 2016; 230: $185-95$.

22 Panov A, Orynbayeva Z. Determination Of Mitochondrial Metabolic Phenotype Through Investigation Of The Intrinsic Inhibition Of Succinate Dehydrogenase. Anal Biochem. 2017; 2697: 30395-0.

23 Paradies G, Ruggiero FM, Dinoi P, et all. Decreased cytochrome oxidase activity and changes in phospholipids in heart mitochondria from hypothyroid rats. Arch Biochem Biophys. 1993; 307: 91-5.

24 Paradies G, Ruggiero FM, Petrosillo G, et all. Enhanced cytochrome oxidase activity and modification of lipids in heart mitochondria from hyperthyroid rats. Biochem Biophys Acta. 1994; 1225: 165-70.

25 Mukherjee S, Samanta L, Roy A, Bhanja S, Chainy GB. Supplementation of $\mathrm{T} 3$ recovers hypothyroid rat liver cells from oxidatively damaged inner mitochondrial membrane leading to apoptosis. Biomed Res Int. 2014: 590897.

26 Carreras MC, Peralta JG, Converso DP, et all. Modulation of liver mitochondrial NOS is implicated in thyroid-dependent regulation of $\mathrm{O}(2)$ uptake. Am J Physiol Heart Circ Physiol. 2001;281:H2282- H22828.

27 Parihar MS, Nazarewicz RR, Kincaid E, et al. Association of mitochondria nitric oxide synthase activity with respiratory chain complex I. Biochem Biophys Res Commun. 2008;366(1):23-28.

28 Weidinger A, Mullebner A, Paier-Pourani J, et all. Vicious inducible nitric oxide synthase-mitochondrial reactive oxygen species cycle accelerates inflammatory response and causes liver injury in rats. Antioxid Redox Signal. 2015; 22: 572-86. 